\title{
Study of variability of 2MASSJ10183905+0014078 stellar objects
}

\author{
E. C. Romas* \\ Observatory ISON-Kislovodsk, Russia
}

\begin{abstract}
This paper presents observational data for the star 2MASSJ10183905+0014078 (UCAC4 452048383), in which so far no brightness variability has been detected. However, photometric observations made in 2015 allowed recording flare activity with an amplitude of $0.8 \mathrm{~m}$ for this star. The star parameters, namely magnitude and color index $\left(\mathrm{V}_{0}=7.59,(\mathrm{~B}-\mathrm{V})_{0}=1.19\right)$ and, therefore, the position on the Hertzsprung-Russell diagram, luminosity $\left(\mathrm{L}_{\odot}=0.1\right)$, spectral class $(\mathrm{K} 7)$, mass $\left(\mathrm{M}_{\odot}=0.6\right)$, the spectral characteristics in the optical $(\mathrm{Ca}, \mathrm{Fe}, \mathrm{Na} \mathrm{D}, \mathrm{H} \alpha$ lines, $\mathrm{Mg} \mathrm{H}$, and $\mathrm{TiO}$ bands) and X-ray ranges allow us to classify the star as UVCet type object. The flash profile can be attributed to type II, with a relatively slow increase and decrease in brightness.
\end{abstract}

Keywords: stars: variability - stars: UV Cet - stars: individual: 2MASSJ10183905 + 001407

\section{Introduction}

Studies of such a phenomenon as stellar flare activity have a long history (Ambartsumyan et al., 1970, Gershberg \& Shakhovskaya, 1973). At first the term "flare stars" has been used to designate dwarf K and M stars with transient optical brightening (UV Ceti stars). Further, improved detector performance and access to new spectral regions have led to detection of flare activity in many other kind of stars, including pre-main and post-main sequence stellar objects. From the point of view of modern concept about the physics of flare activity, it is believed that stellar flares are localized eruptions in magnetic fields, like on the Sun. In some cases, it is possible that we observe atmospheric reactions to mass transfer or other dynamic processes during flares. In all cases, the observations of flare stars never show any doubt that the cause of the observed light curve is the same as on the Sun. However, it cannot be ruled out that only the symptoms are similar, and at different stages of evolution, objects of different spectral types have different flash generation mechanisms (Pettersen, 1989).

A special place among the flare stars is assigned to UVCet-type dwarf stars with late $\mathrm{K}$ - M spectral classes, whose flares are similar to solar, but the energy emitted by them is several orders of magnitude greater than the energy of solar flares. Researchers flash profiles are divided into two main types. I-type flashes are short with a rapid increase in brightness and flashes of II-type, in which the increase and decrease in brightness occurs about 10 times slower (Smirnov, 2015).

This paper presents the observational data for the star 2MASSJ10183905+0014078 (UCAC4452048383), in which so far no brightness variability has been detected. However, the photometric observations made in 2015 made it possible to register flare activity in this star.

\section{Observations and data processing}

The observations of 2MASSJ10183905+0014078 stellar object were performed at the ISON-Kislovodsk Observatory (MPC code D00) in 2015 on the night of March 18-19 during the search for variable

*romasastro@gmail.com 
stars on the GENON telescope (lens diameter $190 \mathrm{~mm}$ and focal length $\mathrm{F}=295 \mathrm{~mm}$ ) with CCD FLIML09000. The resolution of the received images is $8.4 \% /$ pix. On the night of observations, from 18:00:05 UT to 20:20:06 UT, 250 images with an exposure of $30 \mathrm{sec}$, were obtained in the "C" filter $(350 \mathrm{~nm}-900 \mathrm{~nm})$. HWFM of the received images is $9.8 " /$ pix.

Three stars were used as photometric standards:

1) UCAC4-452-048393: V = $12.13 \mathrm{~m}$, color index: 0.46

2) UCAC4-452-048389: V = $13.32 \mathrm{~m}$, color index: 0.84

3) UCAC4-452-048353: V = $11.54 \mathrm{~m}$, color index: 0.92

Initial image processing was performed according to the standard procedure using the MaximDL 5 program. With this program, the stellar magnitudes of objects were also measured. In determining the stellar magnitudes aperture radius, gap radius, and annulus radius were 3 pix, 4 pix and 8 pix, respectively.

In addition to our observations, we also used photometric parameters borrowed from some optical, infrared, as well as X-ray surveys and databases. These include Gaia DR2 (Gaia Collaboration et al., 2018), Fourth U.S. Naval Observatory CCD Astrograph Catalog (UCAC4, Zacharias et al. (2013), Sky Patrol All-Sky Automated Survey for Supernovae (ASAS), LAMOST DR4 catalogs, 2MASS, UKIDSS DR8 LAS (Lawrence et al., 2007), and 3XMM-DR5 (Rosen et al., 2016).

\section{Main parameters}

Fig 1 shows the image of 2MASSJ10183905+0014078 stellar object and Table 1 presents the main parameters of it.

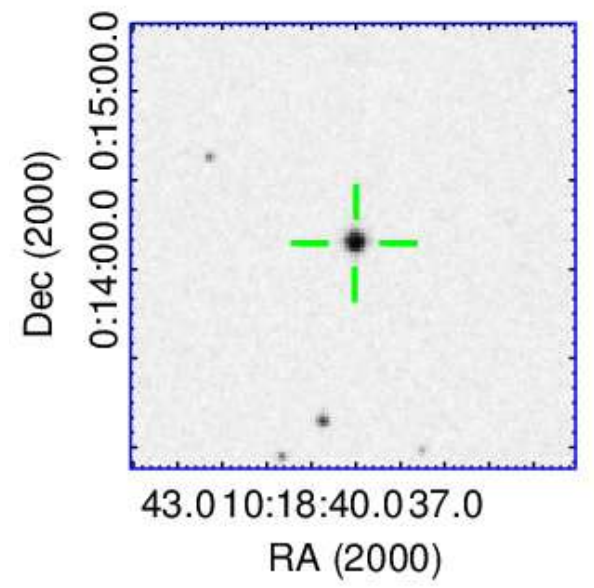

Figure 1. The image of 2MASSJ10183905+0014078 stellar object on the maps of DSS2 R survey.

Table 1. Main parameters of 2MASSJ10183905+0014078

\begin{tabular}{|c|c|c|c|}
\hline \multirow{2}{*}{ UCAC4 } & $\mathbf{B}$ & $\mathbf{V}$ & $\mathbf{R}$ \\
\cline { 2 - 4 } & 14.96 & 13.55 & 13.00 \\
\hline \multirow{2}{*}{ 2MASS } & $\mathbf{J}$ & $\mathbf{H}$ & $\mathbf{K}$ \\
\cline { 2 - 4 } & 10.81 & 10.13 & 9.97 \\
\hline \multirow{2}{*}{ Gaia DR2 } & $\mathbf{P a r}$ & $\mathbf{L}_{\odot}$ & $T_{\text {eff }}$ \\
\cline { 2 - 4 } & 8.9 mas & 0.1 & 4029 \\
\hline
\end{tabular}

According to the parallax value in Table. 1, the distance to the object is $112 \mathrm{pc}$, which corresponds to the distance correction for the absolute magnitude $-5.25 \mathrm{~m}$. Taking into account the value of interstellar absorption $\mathrm{A}_{G}=0.705$, also borrowed from the Gaia DR2 database, then $\mathrm{V}_{0}=7.59$, 
and (according to the law of interstellar absorption Rieke \& Lebofsky $(1985))(\mathrm{B}-\mathrm{V})_{0}=1.19$. This relationship between the luminosity of the star and its color index corresponds to the objects of the Main Sequence, which is reflected in Fig. 2, where our object is marked with a red circle. The photometric data of the near-infrared range also indicate that our stellar object does not exhibit an infrared excess. Fig. 2, borrowed from Pettersen (1989)), shows the Hertzsprung-Russell diagram (G$\mathrm{R}$ ), as well as the position of the flare stars. According to the color indices, the star has a spectral class K7. In the spectrum of the star from the LAMOST DR4 database, the $\mathrm{Ca}, \mathrm{Fe}, \mathrm{NaD}, \mathrm{H} \alpha$ lines, and the $\mathrm{MgH}$, $\mathrm{TiO}$ bands are distinguishable in the range from 4000 to $9000 \AA$ (see Fig. 3).

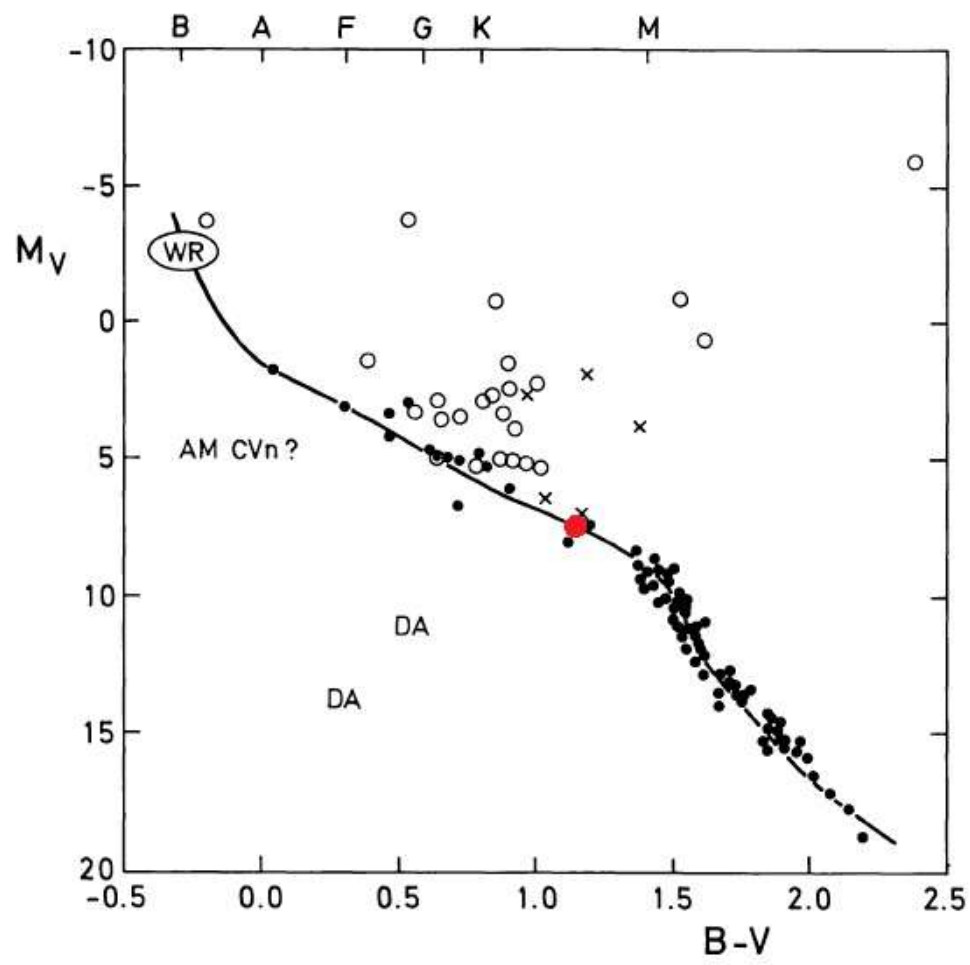

Figure 2. G-R diagram for flaring stars: black circles - flaring stars, crosses - young stars, open circles - giants. The red circle marks the position of 2MASSJ10183905+0014078 object.

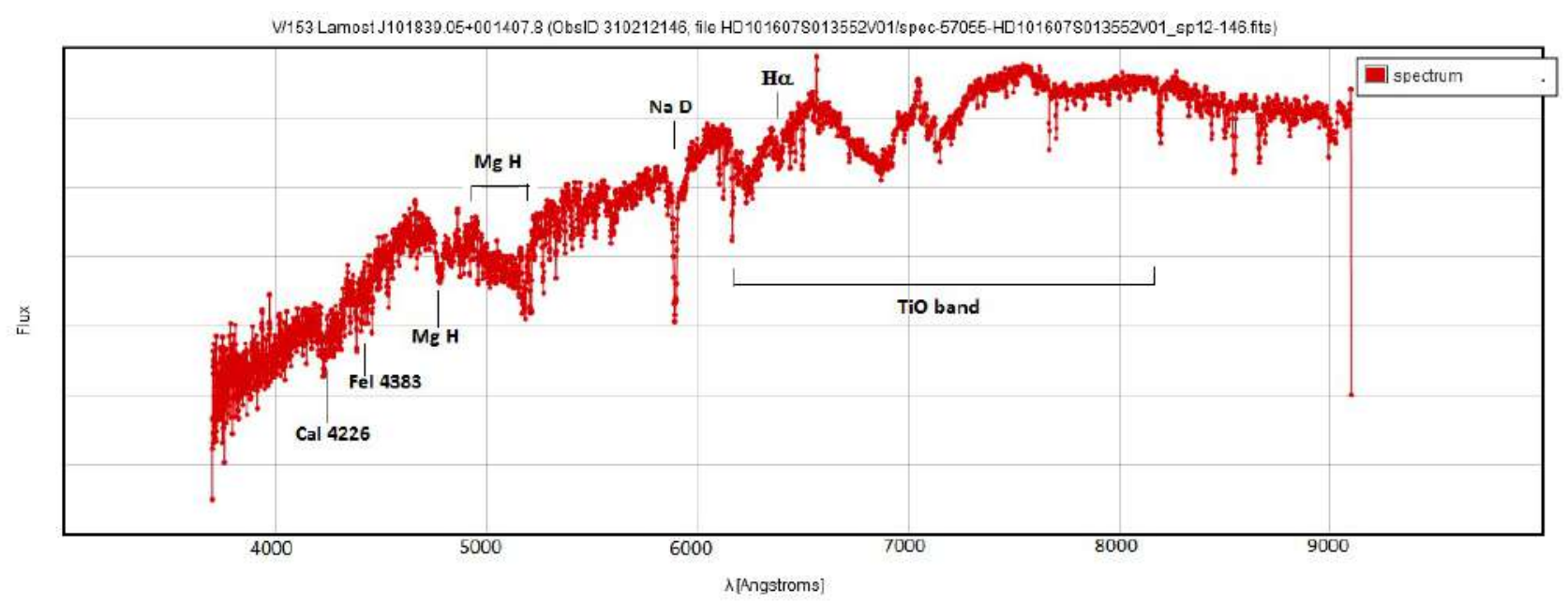

Figure 3. Spectrum of 2MASSJ10183905+0014078 from the LAMOST DR4 catalog.

According to isochrons models from Siess et al. (2000) and conversation tables from Kenyon \& Hartmann (1995) for 2MASSJ10183905+0014078 with the L $\odot$ and $T_{\text {eff }}$ values, which were borrowed 


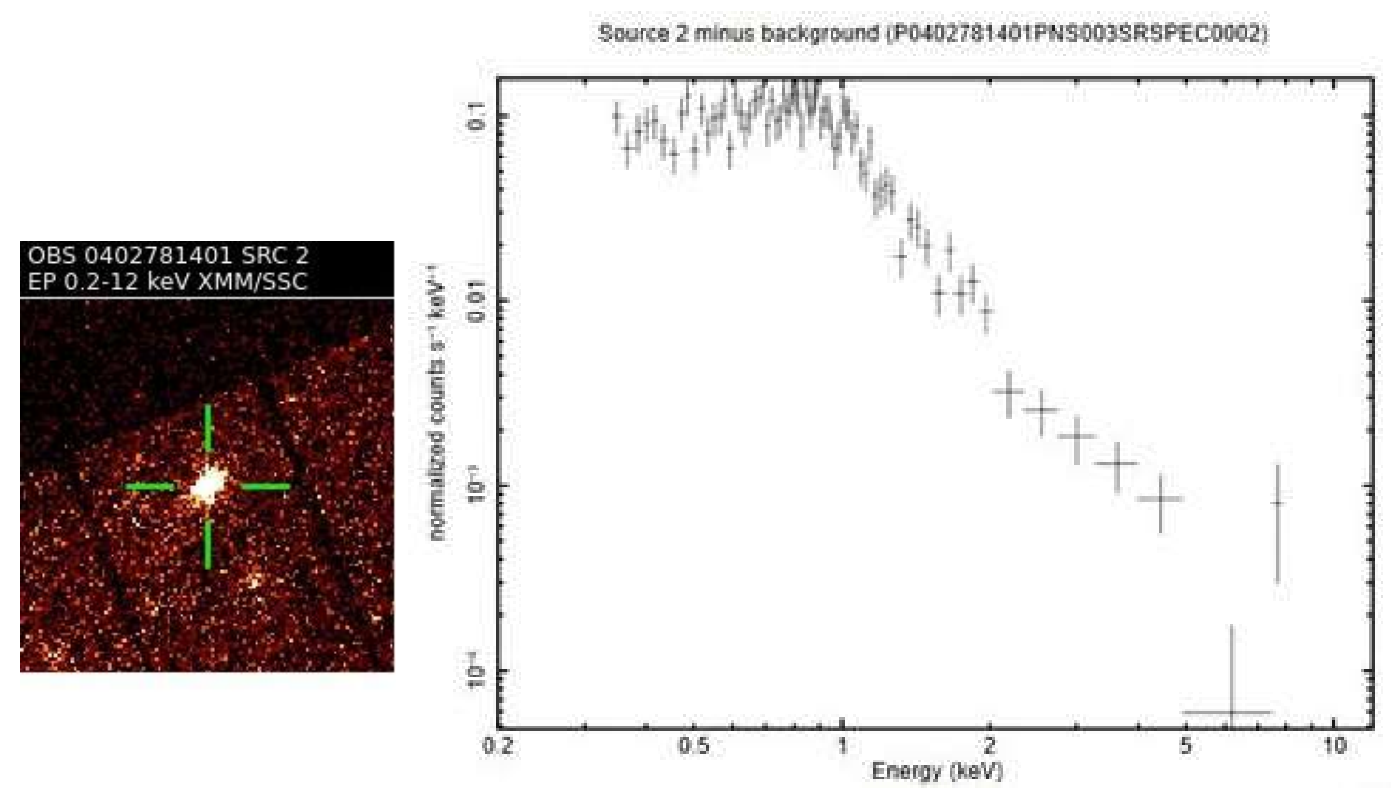

Figure 4. The image (left panel) and the spectrum (right panel) of 2MASSJ10183905 + 0014078 stellar object in the X-ray range from 3XMM-DR5 catalog.

from the Gaia DR2 data base, the mass estimate (for the solar metallicity model $\mathrm{Z}=0.02$ ) is $0.6 \mathrm{M} \odot$, which corresponds to upper mass limit of known UV Cet flare stars (Smirnov, 2015).

Like most flare stars, 2MASSJ10183905+0014078 also shows X-ray activity. On Fig. 4 the image and the spectrum of the object obtained in the X-ray range (3XMM-DR5 catalog) are presented.

\section{Variability of 2MASSJ10183905+0014078}

So far, the star 2MASSJ10183905+0014078 has not been identified as a variable. According to the ASAS data, this star showed only small fluctuations in the brightness of the order of $0.2 \mathrm{~m}$ in the V and g ranges. (see Fig. 5).

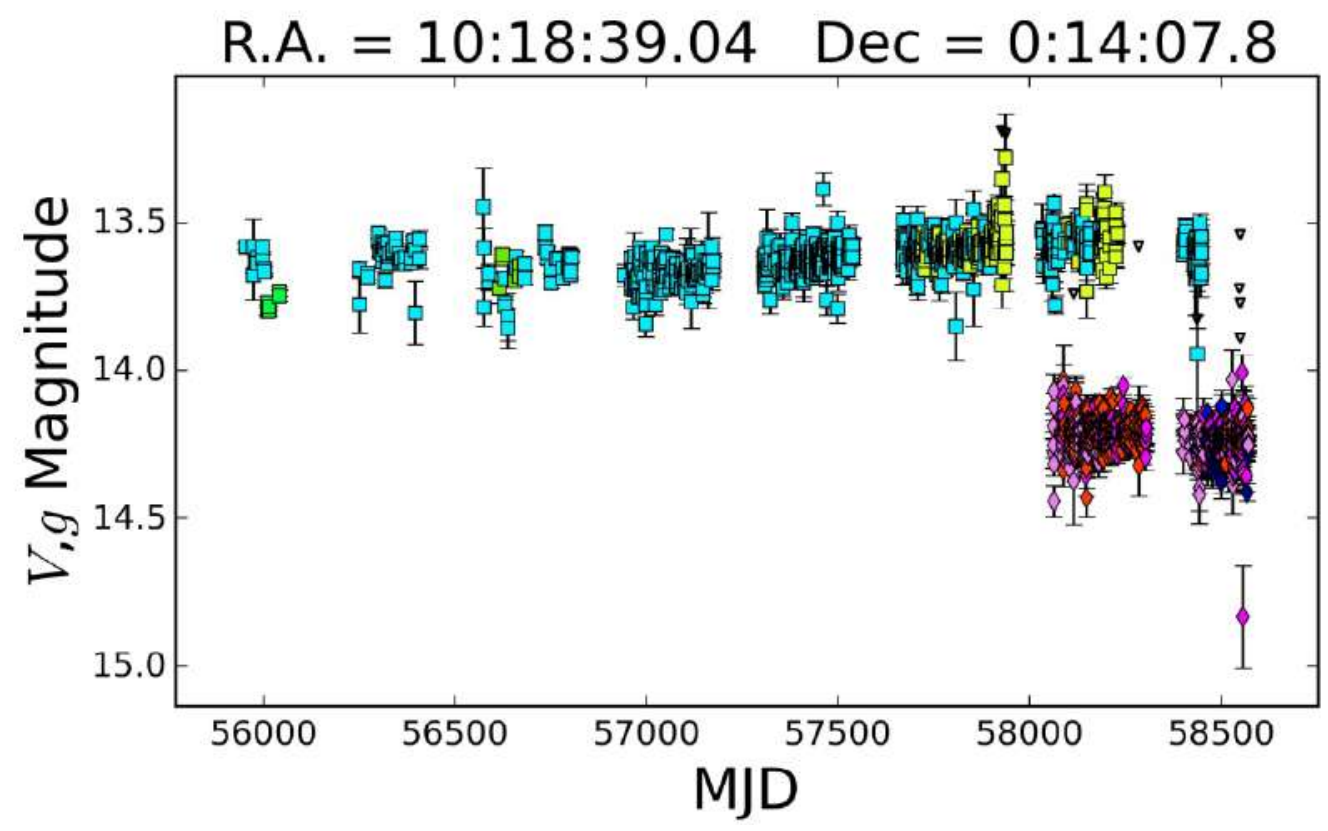

Figure 5. The light curve of 2MASSJ10183905+0014078 from the ASAS database.

However, our observations made on the night of March 18-19, 2015, revealed an outburst of bright- 
ness with amplitude of $0.8 \mathrm{~m}$, which is shown in Fig. 6, where for comparison the light curves of standard stars are given. As can be seen from the data of photometric observations, the light curves of standard stars undergo only minor fluctuations, while the 2MASSJ10183905+0014078 shows a significant increase in brightness and its subsequent decline. Unfortunately, we were unable to continue the observations until the moment when the brightness of the object will drop to its original, basic level. However, according to the existing flash profile, it can be attributed to type II, with a relatively slow increase and decrease in brightness (Smirnov, 2015).

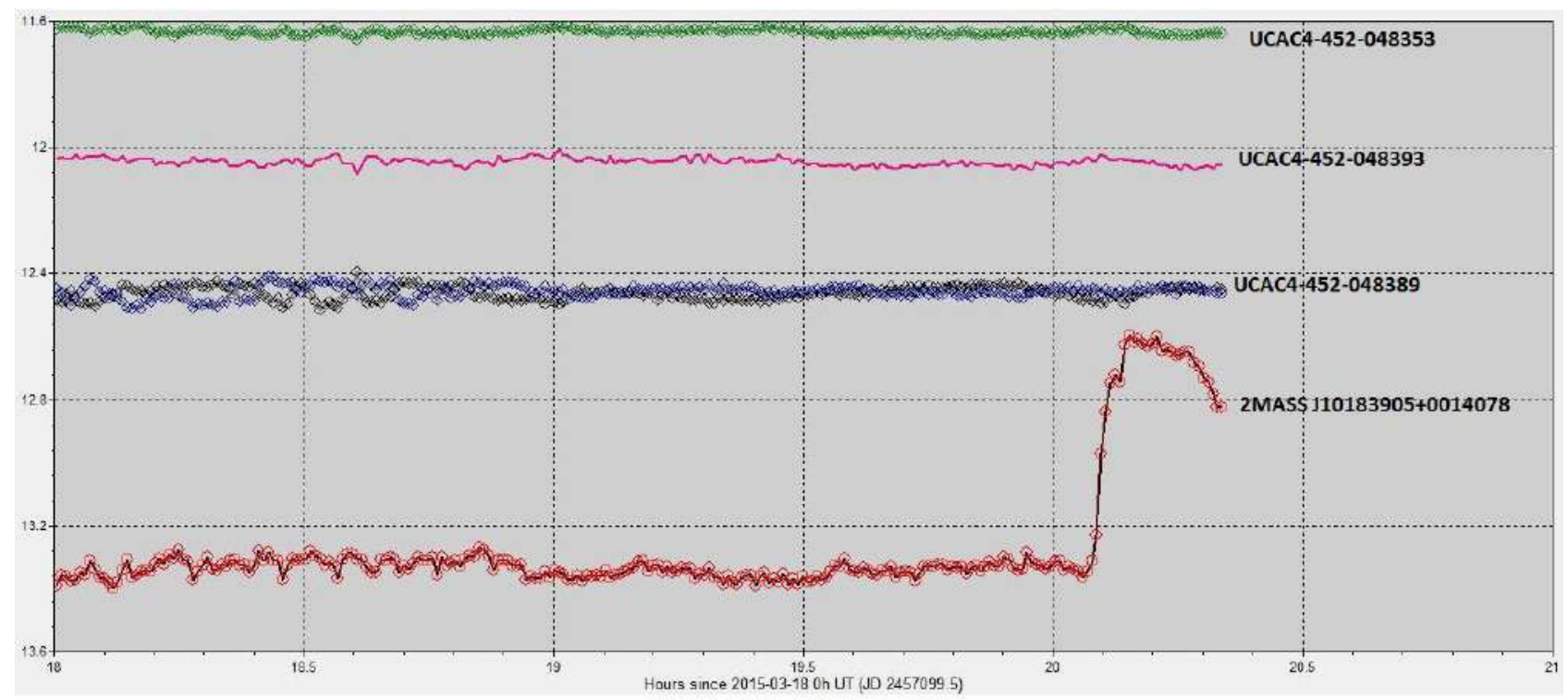

Figure 6. Light curves of 2MASSJ10183905+0014078, UCAC4-452-048353, UCAC4-452-048393, and UCAC4-452-048389 stellar objects, obtained on the night of March 18-19, 2015.

The study of archival data allowed in two other cases to reveal a significant fluctuation in the brightness of this star. First, this is the USNO B1 catalog data, according to which the brightness of a star in the $\mathrm{R}$ range on the maps of the First Palomar Atlas is $\sim 0.6 \mathrm{~m}$ higher than the brightness on the maps of the Second Palomar Atlas (DSS R1 $=12.59 \mathrm{~m}$ and DSS R2 $=13.18 \mathrm{~m}$ ). However, in the B range, no noticeable difference in brightness is observed (DSS B1 $=14.84$ and DSS B2 = 15.02). Secondly, the brightness variability in the near-infrared range. According to the magnitudes of the star in two infrared databases, namely 2MASS (epoch of observation 2000) and UKIDSSD R8 LAS (epoch of observation 2007), the brightness of the object in the $\mathrm{H}$ range also varied within $0.6 \mathrm{~m}$ (UKIDSS $\mathrm{H}=10.77$ ). In the other two bands are not observed significant differences (UKIDSS $\mathrm{J}=$ 11.84 and UKIDSS $\mathrm{K}=10.18$ ).

\section{Discussion and Conclusion}

Thus, photometric observations made on the night of March 18-19 in 2015 revealed a flash of brightness with an amplitude of $0.8 \mathrm{~m}$ in an unregistered until now as a variable 2MASSJ10183905+0014078 (UCAC4 452-048383) star. The star parameters, namely magnitude and color index $\left(\mathrm{V}_{0}=7.59\right.$, (B $\left.-\mathrm{V})_{0}=1.19\right)$ and, therefore, the position on the $\mathrm{G}-\mathrm{R}$ diagram, luminosity $\left(\mathrm{L}_{\odot}=0.1\right)$, spectral class (7), mass $\left(\mathrm{M}_{\odot}=0.6\right)$, the spectral characteristics in optical (Ca, Fe, Na D, H $\alpha$ lines, $\mathrm{Mg} \mathrm{H}$, and $\mathrm{TiO}$ bands) and X-ray ranges allow us to classify the star as UV Cet type object. The flash profile can be attributed to type II, with a relatively slow increase and decrease in brightness. Unfortunately, the observation period does not allow us to fully trace the decline in brightness to the initial level. Undoubtedly, the photometric and spectral studies of this object will be continued.

In conclusion, I would like to note the following. It is known that modeling of stars, even at rest is a challenge, which is still unresolved. And the modeling of flashing stars is important for several reasons. Presumably, flares, including those of the stars of type UV Cet, arise due to the magnetic field of the star. If, based on the parameters of stars, it would be possible to calculate the magnetic field, then it 
would be possible to compare the theory of the generation of a magnetic field and observations. This will undoubtedly greatly help us to fully understand the nature of flare activity. For this reason, the search for new flashing stars or observation of already known ones is of considerable scientific interest.

\section{Acknowledgements}

The author thanks E. Nikoghosyan for his assistance in writing the article.

\section{References}

Ambartsumyan V. A., Mirzoyan L. V., Parsamyan E. S., Chavushyan O. S., Erastova L. K., 1970, Astrophysics, 6, 1

Gaia Collaboration et al., 2018, A\&A, 616, 1

Gershberg R. E., Shakhovskaya N. I., 1973, Nature Physical Science, 242, 85

Kenyon S. J., Hartmann L., 1995, ApS, 101, 117

Lawrence A., et al., 2007, MNRAS, 379, 1599

Pettersen B. R., 1989, Soph, 121, 299

Rieke G. H., Lebofsky M. J., 1985, ApJ, 288, 618

Rosen S. R., et al., 2016, A\&A, 590, 1

Siess L., Dufour E., Forestini M., 2000, A\&A, 358, 593

Smirnov A. A., 2015, "UV Cet type stars", St. Petersburg State University, pp 1-18

Zacharias N., Finch C. T., Girard T. M., Henden A., Bartlett J. L., Monet D. G., Zacharias M. I., 2013, AJ, 145, 44 